\title{
Research on the Pedestrian Evacuation with Arched Congestion
}

\author{
Lei Zhang ${ }^{1, a}$ \\ ${ }^{1}$ School of Traffic and Transportation, Beijing Jiaotong University, Beijing, 100044, China \\ astephen_zhangl@163.com
}

Keywords: Pedestrian push-force; Pedestrian evacuation; Arched congestion; Cell automaton;

Abstract. Based on the panic evacuation of the cell automaton model, we can simulate the flow of people from the behavior of self organization to the phenomenon caused by arched congestion pedestrian flow. Finally, the simulation reproduces the process of pedestrian evacuation during the evacuation, and the formation mechanism of the pedestrian flow during the evacuation.

\section{Introduction}

With a rapid expansion of the urban size and population, the large-scale activities of pedestrian became very frequent. In order to meet the demand of the city dweller for group activities and trips, the building size of pedestrian facilities is increasingly enlarged such as transportation hubs, stadiums, theaters, shopping center. The large pedestrian facilities have some characteristics, such as complex spatial layout, large-term construction cycle, organization difficulty of emergency evacuation and so on. Therefore, pedestrian congestion not only brings great inconvenience, including stadiums and pedestrians in a large gathering of parades, meetings and other group activities, and pedestrians are very intensive, large-scale amusement park and other places of entertainment, but also causes stampede due to pedestrian pushing and so on.[1].

In the research field of evacuation from a large pedestrian model, the work mainly include the evacuation sign[2], evacuation path or exit choice[3], evacuation design and evaluation[4], evacuation simulation[5], evacuation dynamics [6], etc... The theory and method about pedestrian push-force are relatively less researched.

This paper analyzes the generation, absorption, transfer and gather of pedestrian push-force. Initial push-force facing to exit is generated based on the distance between pedestrian and exit. The scalar and vector sum of push-force are introduced to respectively describe the push effect and resultant force of outside jam push-force in crowded evacuation.Absorption coefficient and anti-crush coefficient are introduced to respectively describe the ability for pedestrian to absorb and resist the outside jam push-force.

\section{Push-force Model}

Push-force model is established in the two dimensional cellular grid system, where only one pedestrian is admitted in a cellular. Evacuation space fences and the safety exit constitute the system boundary. Pedestrians can reach the safe exit through lane-changing and over empty cell with no contact force and injury caused by congestion when the pedestrian density is low. Pedestrian residence and congestion will occur at evacuation bottleneck with pedestrian density increasing. Finally, the phenomenon that individual pedestrians holding their positions, pushing around people and rejecting others' jostle will occur because of no moving space and gagging to 
the safe exit, which results in the interaction force and injury caused by congestion.

In the system distance of the cellular safe exit can be described in Eq. 1.

$S_{x y}=\left\{\begin{array}{l}\min _{j}\left(\sqrt{\left(x-x_{j}\right)^{2}+\left(y-y_{j}\right)^{2}}\right. \\ \mathrm{M}\end{array}\right.$

Where, $S_{x y}$ is the distance from cell $(x, y)$ to the safe exit; $\left(x_{j}, y_{j}\right)$ is the $j$ cell in the system; $\mathrm{M}$ is a extremely big number which indicates pedestrian cannot produce $F_{0}$ to the wall.

The push-force F0 is transmitted through pedestrians' interaction contact. Since the static friction of pedestrians and the ground, pedestrian's resilience and push-force interaction, push-force is absorbed and counteracted in the transfer process. Push-force absorption is that the pedestrians or the wall will absorb it in the transfer process and the force is interrupted. Push-force cancellation is that the push-forces in different directions will form resultant forces, which can be cancelled out. The directions of the force will change simultaneously. The transfer rule of the pedestrian Push-force is put forward as shown in Eq. 2.

$$
F_{\text {out }}= \begin{cases}F_{0} & \sum\left|F_{i}\right| \leq \alpha \\ \sum_{i} F_{i}+F_{0} & \sum\left|F_{i}\right|>\alpha\end{cases}
$$

Where, $F_{i}$ is the external push-force of pedestrians, $F_{0}$ is the initial push-force generated by pedestrians, $F_{\text {out }}$ is pedestrian as a medium to pass out of the push-force medium.

\section{Results}

The number of pedestrians, the number of pedestrians and the number of people who are injured by the crowd, and the variation curves of the absorption coefficient is shown in Fig.1.The number of pedestrians, the number of people passing by, and the number of pedestrians and the curves of the variation of the number of people with the injury is shown in Fig.2.

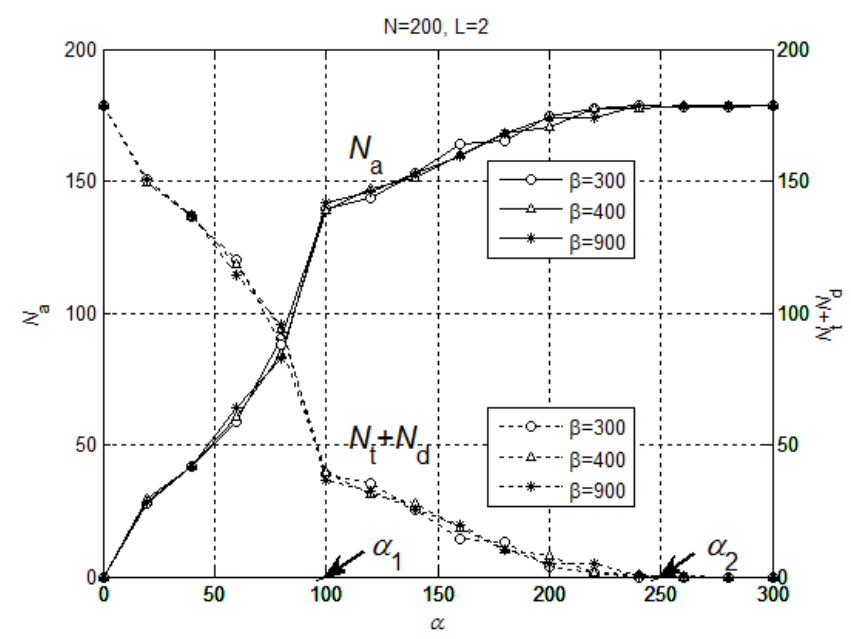

Fig.1. Illustration of the number of pedestrians, the number of pedestrians and the number of people who are injured 


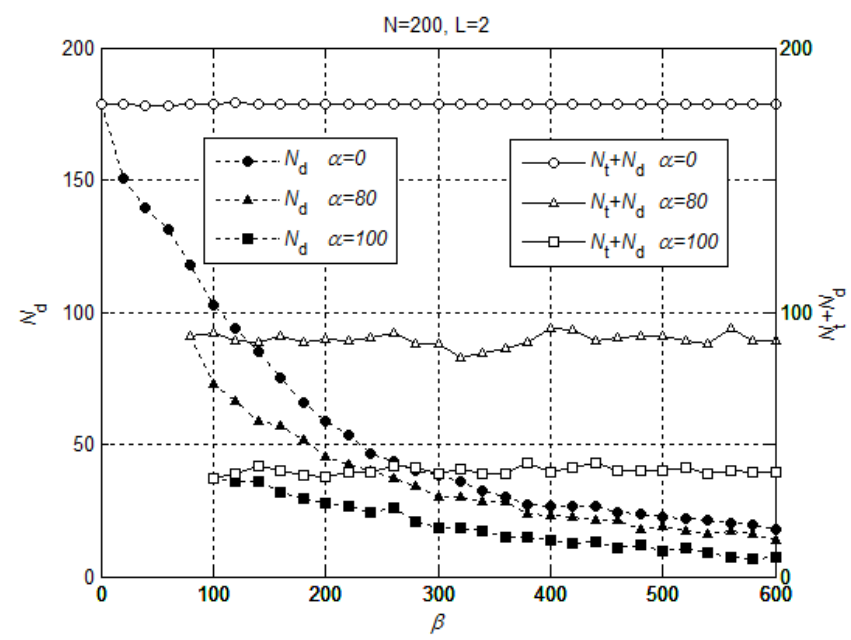

Fig.2. Illustration of he number of pedestrians, the number of people passing by, and the number of pedestrians

The absorption coefficient determines the absorption capacity of the pedestrian to the congestion, and thus determines number of injured pedestrians. The critical coefficient of resistance to death is a weak effect on the prevention of the traffic flow of people. In order to prevent pedestrian traffic jam, in the same time, the absorption coefficient and the absorption capacity of the pedestrian to increase the absorption capacity of the pedestrian are also increased.

\section{Conclusions}

Absorption coefficient and anti-crush coefficient are introduced to respectively describe the ability for pedestrian to absorb and resist the outside jam push-force. Simulation results show that the increase of absorption coefficient or anti-crush coefficient can effectively prevent pedestrian from being injured. It is found that three phases: weak protection, strong protection and complete protection are distinguished based on two critical absorption coefficients and an anti-crush coefficient. Pedestrian casualties will increase with the number of evacuation pedestrian rising. It is also shown that pedestrian casualties in jam occur in a reverse bell-shaped symmetry zone before exit.

\section{Acknowledgment}

Project supported by the Fundamental Research Funds for the Central Universities(2015YJS076).

\section{References}

[1] Helbing D, Farkas I, Vicsek T. Simulating dynamical features of escape panic. 2000.Nature, $\operatorname{Vol}(407)$, p.487.

[2] Helbing D, Farkas I, Vicsek T. Freezing by heating in a driven mesoscopic system. 2000.Physical Review Letters, Vol(84), p.1240.

[3] Song W, Xu X, Wang B H, S Ni. Simulation of evacuation processes using a multi-grid model for pedestrian dynamics. 2006. Physica A, Vol(363), p.492.

[4] Song W G, Yu Y F, Wang B H. Evacuation behaviors at exit in CA model with force essentials: A comparison with social force model. Physica A, 2006, Vol(371), p.658. 
[5] Chen C K, Li J, Zhang D. Study on Evacuation Behaviors at T-shaped Intersection by Force-driving Cellular Automata Model. 2012. Physica A, Vol(391), p.2408.

[6] Guo R Y, Huang H J. A modified floor field cellular automata model for pedestrian evacuation simulation. 2008, Physica A: Math. Theor, Vol(41), p.1.

[7] Kirchner A, Schadschneider A. Simulation of evacuation processes using a bionics-inspired cellular automaton model for pedestrian dynamics. 2002. Physica A, Vol(312), p.260. 